\title{
Identifying barriers to the care of the rheumatoid hand in China: comparing attitudes of rheumatologists and hand surgeons
}

\author{
Lauren E. FRANZBLAU, ${ }^{1}$ Keming WANG ${ }^{2}{ }^{2}$ Xuebing LIANG $^{2}{ }^{2}$ Yang GUO, ${ }^{3}$ Yingni LI, ${ }^{4}$ \\ Yu-chieh (Jay) LIN, ${ }^{5}$ Sunghee LEE, ${ }^{6}$ Beth-Ellen PENNELL, ${ }^{5}$ Melissa J. SHAUVER ${ }^{7}$ and \\ Kevin C. $\mathrm{CHUNG}^{7}$ \\ ${ }^{1}$ University of Michigan Medical School, Ann Arbor, MI, USA, ${ }^{2} 17$ th Department of Plastic Surgery, Plastic Surgery Hospital, \\ Chinese Academy of Medical Sciences, Peking Union Medical College, ${ }^{3}$ Department of Hand Surgery, Jishuitan Hospital, \\ ${ }^{4}$ Department of Rheumatology and Immunology, Peking University People's Hospital, Beijing, China, ${ }^{5}$ Survey Research Center, \\ ${ }^{6}$ Survey Methodology Program, Institute for Social Research, and ${ }^{7}$ Section of Plastic Surgery, University of Michigan, Ann Arbor, MI, \\ USA
}

\begin{abstract}
Aim: In China, hand surgeons treat fewer rheumatoid arthritis (RA) patients compared to other countries. We investigated whether physician and surgeon knowledge, attitudes and practices regarding RA hand deformities reflect current evidence and may contribute to the low utilization of surgery.

Method: We surveyed hand surgeons and rheumatologists at three tertiary hospitals in Beijing, China. Questionnaires were developed from literature and expert review to assess their knowledge, attitudes and practice patterns related to rheumatoid hand surgery.

Results: Thirty-five hand surgeons and 59 rheumatologists completed the survey. Roughly one-third felt that the rheumatologists and hand surgeons agree on how to manage RA hand deformities. One-fifth of rheumatologists and $29 \%$ of hand surgeons believed that drug therapy can correct hand deformities, which contradicts current evidence. Likewise, 30\% and 14\%, respectively, recommended surgery for early-stage hand sequelae that do not meet current indications for surgery. Over $80 \%$ of surgeons and rheumatologists had no exposure to the other specialty during training and felt their training on the treatment of rheumatoid hand deformities was inadequate.

Conclusion: Although we found similar interspeciality disagreement in China as is seen in the United States, there appears to be less interaction through training and consultations. Our results also indicate potential deficits in training and unawareness of evidence and indications for rheumatoid hand surgery. These findings help to explain why surgery for rheumatoid hand deformities is rare in China; doctors have fewer opportunities to collaborate across specialties and may not be able to select appropriate candidates for surgery.
\end{abstract}

Key words: China, hand surgery, rheumatoid arthritis, rheumatology, survey.

Correspondence: Dr Kevin C. Chung, Section of Plastic Surgery, University of Michigan Health System, 2130 Taubman Center, SPC 5340, 1500 E. Medical Center Drive, Ann Arbor, MI, 48109-5340, USA. Email: kecchung@med.umich.edu

\section{INTRODUCTION}

Rheumatoid arthritis (RA) is an autoimmune disease that causes progressive joint deterioration and affects the hands in over $70 \%$ of patients, leading to functional 
impairment, pain and joint deformities. ${ }^{1}$ In the United States, Western Europe and Japan, hand surgery procedures, such as arthroplasty, arthrodesis and tenosynovectomy, are used to treat advanced RA hand deformities with good outcomes. ${ }^{2-5}$ However, in China where estimates of the prevalence of RA range from $0.28 \%$ to $0.45 \%,{ }^{6-8}$ these reconstructive procedures are rarely performed. ${ }^{5}$ This anomalous management of RA hand deformities has not been explained, but may be related to disagreement among specialists and practice patterns that are not fully engaged in current evidencebased medicine.

Metacarpophalangeal (MCP) joint arthroplasty is a common hand surgery procedure among RA patients. ${ }^{3,4,9}$ Long-term prospective outcome investigations support its ability to reduce hand pain, increase hand function, correct ulnar drift and achieve favorable aesthetic results in patients with advanced joint disease. ${ }^{4,11,12}$ Yet prior studies in the United States demonstrate marked disagreement between rheumatologists and hand surgeons about the indications for surgery, and variability in practice patterns that can affect referral for surgery. ${ }^{13}$ Although these same challenges may face Chinese doctors, it is likely that other factors, such as insufficient knowledge and training, prohibit greater use of RA hand surgery in China. Among Chinese physicians who treat cancer patients, for example, misconceptions and lack of knowledge prevent the proper use of morphine for cancer pain management, which hampers patients' quality of life. ${ }^{14}$ RA joint deformities are a multidisciplinary problem best managed by a team of rheumatologists and surgeons. ${ }^{15-17}$ All doctors involved must be well versed in the available treatment options and indications to practice evidence-based medicine and to appropriately counsel patients.

We undertook a pilot study of hand surgeons and rheumatologists at three tertiary academic health centers in Beijing to explore how each specialty approaches RA hand deformities. Specifically we sought to understand the cause for the low rate of RA hand surgery in China by investigating the following: rheumatologist and hand surgeon training and practices; knowledge of rheumatoid hand deformities; perceptions of the other specialty; and response to two clinical scenarios to gauge the knowledge of surgical indications. Using these data, we compared Chinese rheumatologists and hand surgeons to assess whether interspeciality disagreement is present and whether self-reported knowledge and practice patterns reflect current evidence. We hypothesize that the lack of interdisciplinary collaboration and understanding of evidence-based surgical indications and outcomes contribute to the low rate of RA hand surgery in China.

\section{METHODS}

Our Institutional Review Board approved this methodology.

\section{Sample}

We obtained convenience samples of rheumatologists and hand surgeons from three large teaching hospitals in Beijing during the summer of 2015. All three sites are prominent institutions in Beijing and nationally with expertise in internal medicine, scientific research and hand surgery. Rheumatologists and hand surgeons were eligible if they were actively practicing in China and had completed medical school (i.e., Bachelors, Masters or Doctoral degrees in medicine) and residency training either in internal medicine or in general, orthopedic or plastic surgery. Completion of fellowship training was not a requirement. In addition to staff doctors at these three hospitals, we also invited visiting professors and attendees from one morning session of a national rheumatology conference at one of the sites to participate.

\section{Questionnaires}

We developed questionnaires in English based on review of the relevant literature as well as input from clinical and methodological collaborators from the United States and China. Many items were adapted from previous surveys. ${ }^{5-8,10,11,13,16,18-25}$ Our survey instruments captured demographic information, attitudes toward hand surgery, perceptions of the other specialty, and recommendations regarding surgical intervention in two clinical scenarios. Both clinical cases (Appendix 1) focused on MCP joint arthroplasty because it is recognized as a mainstay of reconstruction for rheumatoid joint deformities and there is ample evidence for its indications and outcomes. Clinical indications include radiographic findings of MCP joint destruction or dislocation, palmar subluxation, ulnar deviation and interphalangeal joint stiffness. ${ }^{4,9,15,19}$ Case 1 did not meet these criteria, whereas Case 2 was a candidate for MCP joint arthroplasty.

Expert review by clinical and methodological collaborators from the United States and China was performed to evaluate the content, wording and structure of the surveys. ${ }^{26,27}$ Two different researchers then translated the surveys into Chinese and back-translated them to English. A third compared the back-translation to the 


\begin{tabular}{lccc}
\hline & $\begin{array}{c}\text { Rheumatologists } \\
(n=59)\end{array}$ & $\begin{array}{c}\text { Hand surgeons } \\
(n=35)\end{array}$ & $P$-value \\
\hline Mean age (range) & $37.5(28-60)$ & $42.5(26-60)$ & 0.01 \\
Female & $66 \%$ & $14 \%$ & $<0.0001$ \\
Male & $34 \%$ & $86 \%$ & 0.12 \\
Doctoral (MD) degree & $36 \%$ & $49 \%$ & $<0.0001$ \\
Specialty fellowship & $25 \%$ & $83 \%$ & 0.88 \\
Medical training abroad & $5 \%$ & $6 \%$ & 0.005 \\
Years in practice & & & \\
$<5$ years & $29 \%$ & $14 \%$ & $<0.0001$ \\
$5-9$ years & $44 \%$ & $26 \%$ & \\
10-19 years & $17 \%$ & $20 \%$ & \\
$>$ 20 years & $10 \%$ & $37 \%$ & \\
Trained with the other specialty & $5 \%$ & $17 \%$ & \\
Practice devoted to RA patients & & & \\
$0 \%$ & $0 \%$ & $6 \%$ & \\
$<25 \%$ & $7 \%$ & $91 \%$ & \\
$25-49 \%$ & $59 \%$ & $3 \%$ & \\
$50-74 \%$ & $29 \%$ & $0 \%$ & \\
$75-99 \%$ & $3 \%$ & $0 \%$ & \\
$100 \%$ & $2 \%$ & & \\
\hline
\end{tabular}

Table 1 Respondent characteristics

original English. ${ }^{22}$ This stepwise method of translation was used to ensure the accuracy of the survey content. We revised the Chinese surveys to address any differences between the original and back-translated versions. Then clinical collaborators from the Beijing Hospitals reviewed both questionnaires for content and language. The final instruments had 26 items for rheumatologists and 31 for hand surgeons, and took about $10 \mathrm{~min}$ to complete.

\section{Data collection}

Rheumatologists and surgeons were recruited from three hospitals. We approached clinicians before or after their clinics at two sites, and at the third, rheumatologists were surveyed at a departmental meeting. In addition, we distributed surveys to all doctors attending a morning session of the national conference. These surveys were collected at the end of the presentations. Surveys were exempt from written informed consent because participation was restricted to voluntarily completing an anonymous survey. Each participant was offered a pen for participating.

\section{Data analysis}

Data were organized by medical specialty and descriptive statistics were calculated for questionnaire responses and demographic data. We compared responses between specialty groups using Wilcoxon-
Mann-Whitney tests for continuous variables and Chi-square tests or Fisher exact tests for categorical variables.

\section{RESULTS}

Fifty-nine rheumatologists representing 38 hospitals from 19 provinces completed the questionnaires. Thirty-four hand surgeons from Beijing, as well as one from Taiwan participated. All physicians and surgeons (100\%) approached in the clinic agreed to participate in the study. From the rheumatology conference, 48 rheumatologists were invited to participate. Thirty-six (75\%) returned completed surveys, of which 25 met our eligibility criteria (i.e., had completed their residency training). In our sample, hand surgeons were older, had been in practice longer, and were more likely to be male than rheumatologists; $5 \%$ of rheumatologists and $17 \%$ of hand surgeons had trained with members of the other specialty. Only five doctors had received training outside of mainland China, including in England and Taiwan. Detailed respondent characteristics are shown in Table 1.

\section{Attitudes toward management of rheumatoid hand deformities}

Most rheumatologists (81\%) and hand surgeons (80\%) estimated that hand deformities occur in over $50 \%$ of 
Table 2 Attitudes toward management of rheumatoid hand deformities

\begin{tabular}{|c|c|c|c|}
\hline & Rheumatologists $(n=59)$ & Hand surgeons $(n=35)$ & $P$-value \\
\hline Satisfied with ability to manage RA hand deformities & $41 \%$ & $62 \%$ & 0.04 \\
\hline $\begin{array}{l}\text { Feel that training received in management of RA hand } \\
\text { deformities was adequate }\end{array}$ & $10 \%$ & $17 \%$ & 0.35 \\
\hline Agree that drugs can correct hand deformities & $20 \%$ & $29 \%$ & 0.21 \\
\hline Agree that surgery can correct RA hand deformities & $92 \%$ & $100 \%$ & 0.007 \\
\hline $\begin{array}{l}\text { Feel there is good or excellent evidence supporting } \\
\text { metacarpophalangeal arthroplasty for RA hand deformities }\end{array}$ & $14 \%$ & $49 \%$ & $<0.0001$ \\
\hline
\end{tabular}

RA patients; prior research indicates the prevalence is around $70 \% .{ }^{1}$ Nearly all respondents agreed that surgery can correct RA hand deformities (Table 2), except for $8 \%$ of rheumatologists (compared to $0 \%$ of hand surgeons, $P=0.007$ ). Hand surgeons were significantly more likely to endorse the availability of good or excellent evidence supporting MCP joint arthroplasty (49\% vs. $14 \%, P<0.0001)$ and to be satisfied with their ability to treat RA hand deformities $(62 \%$ vs. $41 \%$, $P=0.04)$. Despite this satisfaction only a minority of rheumatologists (10\%) and hand surgeons (17\%) felt that they had received adequate training in this area.

\section{Attitudes toward the other specialty}

Roughly one-third of participants felt that rheumatologists and hand surgeons agree on how to manage rheumatoid hand sequelae and less than one-half felt that the other specialty refers/operates at the appropriate time (Table 3 ). Hand surgeons were less likely to report positive views of rheumatologists' management than rheumatologists of surgeons' (40\% vs. 53\%, $P=0.07)$.

\section{Response to clinical cases}

Specialists responded differently to the clinical cases (Appendix 1), which included early (Stage 1) hand involvement with swan-neck and Boutonnière deformities (Case 1) and advanced joint disease (Stage 3) with ulnar deviation and moderate deterioration of articular cartilage (Case 2). Thirty percent of rheumatologists recommended MCP arthroplasty in Case 1 compared to $14 \%$ of hand surgeons $(P=0.08)$. There was no difference for Case $2(95 \%$ vs. $91 \%, P=0.50)$.

\section{DISCUSSION}

To assess knowledge and attitudes regarding hand surgery for RA in China, we performed a pilot survey of 59 rheumatologists and 35 hand surgeons at three large tertiary hospitals in Beijing. Our results show that few doctors trained with members of the other specialty, yet more than half report regular consultations to each other about RA patients. Nearly all respondents agreed that surgery can correct RA hand deformities. However, a subset also indicated that drug therapy can do the same (which it cannot). Surgeons and rheumatologists disagreed about the availability of good or excellent evidence supporting the use of MCP arthroplasty for RA, and differed in their recommendations for surgical treatment for early-stage RA hand deformities. Most respondents indicated that their training on this topic was insufficient. Although the interspeciality

Table 3 Attitudes toward the other specialty

\begin{tabular}{lcc}
\hline & $\begin{array}{c}\text { Rheumatologists } \\
(n=59)\end{array}$ & $\begin{array}{c}\text { Hand surgeons } \\
(n=35)\end{array}$ \\
\hline $\begin{array}{l}\text { Feel that rheumatologists and hand surgeons agree on the management } \\
\text { of rheumatoid arthritis patients' hand disabilities. }\end{array}$ & $31 \%$ & $37 \%$ \\
$\begin{array}{l}\text { Feel that members of other specialty appropriately manage RA } \\
\text { hand deformities }\end{array}$ & $53 \%$ & $40 \%$ \\
$\begin{array}{l}\text { Feel that hand surgeons operate on RA patients at the appropriate } \\
\text { time/rheumatologists refer RA patients at the appropriate time }\end{array}$ & $40 \%$ & $46 \%$ \\
$\begin{array}{l}\text { Feel that hand surgeons are too aggressive in treating RA } \\
\text { patients/rheumatologists are too passive in treating RA patients }\end{array}$ & $40 \%$ & $57 \%$ \\
$\begin{array}{l}\text { Regularly consult with a hand surgeon/rheumatologist about } \\
\text { my RA patients' treatments }\end{array}$ & $58 \%$ & $57 \%$ \\
\hline
\end{tabular}


discrepancies are similar to controversies found in the United States, our results also suggest that doctors in China are not completely aware of indications for hand surgery or its benefits compared to drug therapy for advanced RA joint sequelae.

Despite reporting similar attitudes toward each other as have been found in American rheumatologists and hand surgeons, doctors in our study reported less interdisciplinary training and active collaboration about RA patients compared to those in the United States. ${ }^{16}$ Alderman et al. ${ }^{13}$ found that $33 \%$ of hand surgeons and $21 \%$ of rheumatologists in the United States were exposed to the other specialty during training, and $78 \%$ and $81 \%$, respectively, have an active referral exchange with the other specialty. Only $17 \%$ of surgeons and $5 \%$ of rheumatologists in this study reported training with each other and fewer $(57 \%, 58 \%)$ reported regularly consulting with the other specialty. This may contribute to the lower rates of rheumatoid hand surgery observed in China compared to the United States, as coordination is key for operative management of RA hand deformities. Careful selection of patients and planning are necessary for hand surgery, a local intervention, to be applied correctly within the context of a systemic disease.

Our data suggest an insufficient knowledge of current evidence regarding rheumatoid hand surgery among rheumatologists and hand surgeons in China, which is concerning because it could preclude judicious applications of procedures such as MCP joint arthroplasty. First, $20 \%$ of rheumatologists and $29 \%$ of hand surgeons believed that drug therapy can correct RA hand deformities. Medication management is indispensable for controlling RA, but it cannot alter hand deformities or joint destruction once these sequelae have occurred. Second, $30 \%$ of rheumatologists and $14 \%$ of hand surgeons recommended MCP arthroplasty in the first clinical scenario (Stage 1 RA), which does not meet established clinical indications for this intervention. Lastly, only $10 \%$ of rheumatologists and $17 \%$ of hand surgeons felt that they received adequate training in the management of RA hand deformities. This implies that doctors, particularly non-surgeons, are not fully prepared to treat RA hand deformities or are familiar with literature about their management. This may impair their ability to make appropriate referrals and could translate into misconceptions among patients who rely on rheumatologists to guide RA treatment decisions. Hand surgeons must also be aware of when and if to intervene, as patient selection is very important to achieving good outcomes. ${ }^{15}$
Several limitations apply to this study. Our samples were modest and collected from one city. Beijing and other large cities in China, such as Shanghai, have advanced medical care that may not be available in smaller cities where barriers related to low knowledge of indications and evidence are likely to be even more prevalent. To enhance geographic diversity, we included doctors visiting from other provinces. Next, we asked participants to self-report attitudes and knowledge, which may not directly reflect practice patterns. Lastly, the appropriateness of reconstruction for RA hand deformities is subject to other factors that were not addressed in this study, such as the patients' needs and preferences, comorbidities, acclimation to hand symptoms and disease control. ${ }^{15}$ However, the scope of this study was to assess clinicians' knowledge of surgical indications, which we were able to capture by asking whether or not they would recommend intervention in specific clinical scenarios.

The findings from this survey of 94 rheumatologists and hand surgeons highlight the issue of low rates of rheumatoid hand surgery in China and help to illuminate clinician-related factors that contribute. Namely, it uncovered interspeciality disagreements and inadequacies in training that could hurt collaborative relationships and limit clinical expertise, as well as recommendations and beliefs about the treatment of RA hand deformities that do not adhere to current evidence. These issues create barriers to RA patient care and rheumatoid hand surgery. Through highlighting them, we hope to encourage and broaden access to evidence-based management of rheumatoid hand conditions in China.

\section{ACKNOWLEDGEMENTS}

Research reported in this publication was supported by the National Institute of Arthritis and Musculoskeletal and Skin Diseases of the National Institutes of Health under Award Number 2 K24-AR053120-06. The content is solely the responsibility of the authors and does not necessarily represent the official views of the National Institutes of Health.

\section{REFERENCES}

1 de la Mata LJ, Palacios Carvajal J (1998) Rheumatoid arthritis: are outcomes better with medical or surgical management? Orthopedics 21, 1085-6.

2 Anderson RJ (2011) Controversy in the surgical treatment of the rheumatoid hand. Hand Clin 27 (1), 21-5. 
3 Chim HW, Reese SK, Toomey SN, Moran SL (2014) Update on the surgical treatment for rheumatoid arthritis of the wrist and hand. J Hand Ther 27 (2), 134-41; quiz 42.

4 Goldfarb CA, Dovan TT (2006) Rheumatoid arthritis: silicone metacarpophalangeal joint arthroplasty indications, technique, and outcomes. Hand Clin 22, 177-82.

5 Kotsis SV, Chung KC (2005) A qualitative assessment of rheumatoid hand surgery in various regions of the world. J Hand Surg 30, 649-57.

6 Xu C, Wang X, Mu R et al. (2014) Societal costs of rheumatoid arthritis in China: a hospital-based cross-sectional study. Arthritis Care Res 66, 523-31.

7 Zeng QY, Chen R, Darmawan J et al. (2008) Rheumatic diseases in China. Arthritis Res Ther 10 (1), R17.

8 Li R, Sun J, Ren LM et al. (2012) Epidemiology of eight common rheumatic diseases in China: a large-scale crosssectional survey in Beijing. Rheumatology 51, 721-9.

9 Moran SL, Bishop AT (2007) Clinical update: surgical management of rheumatoid hand. Lancet 370, 372-4.

10 Zhao S, Chen Y, Chen H (2015) Sociodemographic factors associated with functional disability in outpatients with rheumatoid arthritis in Southwest China. Clin Rheumatol 34, 845-51.

11 Chung KC, Nellans KW, Burns PB et al. (2015) Patient expectations and long-term outcomes in rheumatoid arthritis patients: results from the SARA (Silicone Arthroplasty in Rheumatoid Arthritis) study. Clin Rheumatol 34, 641-51.

12 Wolfe F, Zwillich SH (1998) The long-term outcomes of rheumatoid arthritis: a 23-year prospective, longitudinal study of total joint replacement and its predictors in 1,600 patients with rheumatoid arthritis. Arthritis Rheum 41, 1072-82.

13 Alderman AK, Ubel PA, Kim HM, Fox DA, Chung KC (2003) Surgical management of the rheumatoid hand: consensus and controversy among rheumatologists and hand surgeons. J Rheumatol 30, 1464-72.

14 Yanjun S, Changli W, Ling W et al. (2010) A survey on physician knowledge and attitudes towards clinical use of morphine for cancer pain treatment in China. Support Care Cancer 18, 1455-60.

15 Chung KC, Pushman AG (2011) Current concepts in the management of the rheumatoid hand. J Hand Surg 36, 736-47; quiz 47.

16 Alderman AK, Chung KC, Kim HM, Fox DA, Ubel PA (2003) Effectiveness of rheumatoid hand surgery: contrasting perceptions of hand surgeons and rheumatologists. J Hand Surg 28 (1), 3-11; discussion 2-3.

17 Siddle HJ, Backhouse MR, Monkhouse R, Harris NJ, Helliwell PS (2011) Joint orthopaedic and rheumatology clinics: evidence to support the guidelines. Musculoskelet Care 9, 180-3.

18 Cheung KW, Chung SL, Chung KY, Chiu KH (2013) Patient perception and knowledge on total joint replacement surgery. Hong Kong Med J 19 (1), 33-7.
19 Chung KC, Burns PB, Wilgis EF et al. (2009) A multicenter clinical trial in rheumatoid arthritis comparing silicone metacarpophalangeal joint arthroplasty with medical treatment. J Hand Surg 34, 815-23.

20 Zhang P, Li J, Han Y, Yu XW, Qin L (2010) Traditional Chinese medicine in the treatment of rheumatoid arthritis: a general review. Rheumatol Int 30, 713-8.

21 Lv QW, Zhang W, Shi Q et al. (2015) Comparison of Tripterygium wilfordii Hook $\mathrm{F}$ with methotrexate in the treatment of active rheumatoid arthritis (TRIFRA): a randomised, controlled clinical trial. Ann Rheum Dis 74, 1078-86.

22 Wang XS, Tang JY, Zhao M, Guo H, Mendoza T, Cleeland CS (2003) Pediatric cancer pain management practices and attitudes in China. J Pain Sympt Manag 26, 748-59.

23 Feng Z, Zhan HJ, Feng X, Liu C, Sun M, Mor V (2011) An industry in the making: the emergence of institutional elder care in urban china. J Am Geriat Soc 59, 738-44.

24 Chung KC, Kotsis SV, Kim HM, Burke FD, Wilgis EF (2006) Reasons why rheumatoid arthritis patients seek surgical treatment for hand deformities. J Hand Surg 31, 289-94.

25 Yip WC, Hsiao WC, Chen W, Hu S, Ma J, Maynard A (2012) Early appraisal of China's huge and complex health-care reforms. Lancet 379, 833-42.

26 Survey Research Center (2016). Guidelines for Best Practice in Cross-Cultural Surveys. Ann Arbor, MI: Survey Research Center, Institute for Social Research, University of Michigan. Retrieved October 1, 2015, from http://www.ccsg.isr.umich.edu/.

27 Groves RM (2009) Survey Methodology, 2nd edn, 461 pp. Wiley, Hoboken, NJ.

\section{Appendix 1: Clinical Cases}

Case 1: A 60-year-old woman with rheumatoid arthritis who is otherwise healthy presents to you for treatment. She has synovitis at the metacarpophalangeal joints, minimal ulnar deviation, and little extension lag of the fingers. She does not complain of pain and has little trouble completing daily tasks except when her joints are swollen in the morning (see picture below).

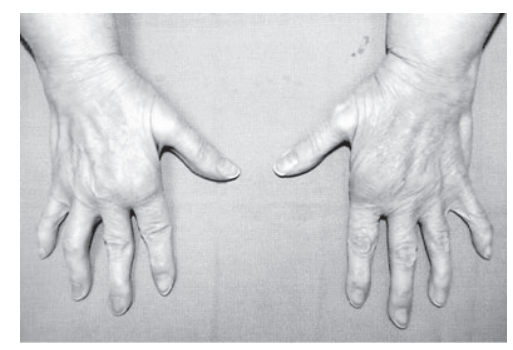

In your clinical opinion, would this patient benefit from metacarpophalangeal arthroplasty? 
a. Yes

b. No

If she would benefit from metacarpophalangeal arthroplasty, how soon should she have it? Leave blank if you answered 'No' to the previous question.
a. As soon as possible
b. During the next 6 months
c. During the next year
d. More than 1 year from now

Case 2: A 60-year-old woman with rheumatoid arthritis who is otherwise healthy presents to you for treatment. Her radiographs show moderate erosion of the articular cartilage. She does not complain of pain but has difficulty completing some activities of daily living (such as doing up buttons or holding a glass) because of her ulnar deviation and the inability to flex her fingers wide and grasp onto objects. (See picture below).

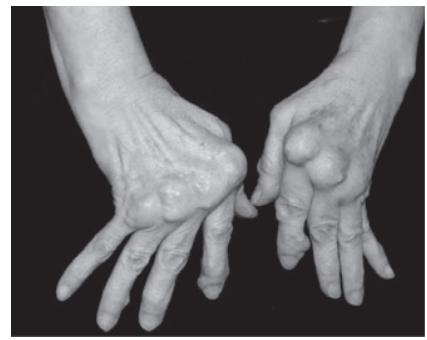

In your clinical opinion, would this patient benefit from metacarpophalangeal arthroplasty?

a. Yes

b. No

If she would benefit from metacarpophalangeal arthroplasty, how soon should she have it? Leave blank if you answered 'No' to the previous question.
a. As soon as possible
b. During the next 6 months
c. During the next year
d. More than 1 year from now 